\title{
In Vitro Dissolution Similarity as a Surrogate for In Vivo Bioavailability and Therapeutic Equivalence
}

\author{
Sally A. Helmy ${ }^{1,2, *}$ and Heba M. El Bedaiwy ${ }^{1}$ \\ ${ }_{1}^{1}$ Department of Pharmaceutics, Faculty of Pharmacy, Damanhour University, Damanhour, Egypt \\ 2 Department of Clinical and Hospital Pharmacy, Faculty of Pharmacy, Taibah University, \\ AL-Madinah AL-Munawarah, Kingdom of Saudi Arabia
}

e-mail: Shelmy@taibahu.edu.sa; Sallyhelmy@yahoo.com

\section{ABSTRACT}

Generic immediate-release solid oral dosage forms containing BCS Class I and III drugs that have similar in vitro dissolution profiles might receive market authorization without in vivo bioequivalence testing. The objective was to investigate whether the different immediate-release generic products containing BCS Class I drugs (fluoxetine and linezolid) and a Class III drug (fluconazole) fulfill the requirements of $85 \%$ or more drug release in 15 or 30 min in all three buffers $(\mathrm{pH}$ 1.2, $\mathrm{pH} 4.5$, and $\mathrm{pH}$ 6.8) using in vitro dissolution testing in accordance with WHO requirements. The dissolution profiles were compared statistically to the relevant reference product by calculating the fit factors $\left(f_{1}\right.$ and $f_{2}$ ), dissolution efficiencies, and mean dissolution time. The amount of drug dissolved in all dissolution media was determined by a validated UV spectrophotometric method. All investigated products of fluoxetine and linezolid met the biowaiver criteria for BCS Class I drugs and would be considered in vitro equivalent. Only one product of the BCS Class III drug fluconazole did not pass the WHO guideline for in vitro equivalence; however, all the products released fluconazole satisfactorily, with at least $80 \%$ dissolved within 30 min. Pharmaceutical equivalence together with in vitro dissolution similarity could be considered suitable to ensure in vivo bioequivalence and, hence, therapeutic equivalence of BCS Class I and III drugs. Not all generic products containing the same drug (BCS Class I or III) in similar strengths and dosage forms are in vitro equivalent.

KEYWORDS: BCS; generic drug; in vitro dissolution similarity; immediate-release solid oral dosage forms; therapeutic equivalence; dissolution.

\section{INTRODUCTION}

G eneric drugs should be pharmaceutically, biologically, and therapeutically equivalent to the reference drug to guarantee interchangeability for pharmacotherapy (1). Drug products are considered therapeutically equivalent if they meet the regulatory criteria of pharmaceutical equivalence and bioequivalence (2-6). Generic pharmaceutical equivalents should contain the same active ingredient(s) manufactured in the same dosage form and administered in the same pathway. Additionally, they should meet the same compendial or other applicable standards and be similar in strength or concentration (1). However, they may differ in shape, excipients, release mechanisms, packaging, scoring configuration, and expiration time (7). Bioequivalence of pharmaceutically equivalent products also implies their therapeutic equivalence (8). In accordance with WHO recommendations $(1,7)$, bioequivalence can be determined by in vivo pharmacokinetic methods and, in some cases, by in vitro dissolution test. A biowaiver is a simplification and reduction of time required for product approval, thus releasing new qualified products to the market at lower costs $(5,9)$. According to the
WHO (3) and the EMA (2), a biowaiver implies that in vivo bioequivalence studies may be substituted by performing in vitro dissolution testing to compare the test product against a reference product.

The Biopharmaceutics Classification System (BCS) differentiates active pharmaceutical ingredients (API) into four different classes according to their solubility and permeability (10). In a science-based approach, BCS allows waiver of in vivo bioavailability and bioequivalence testing for immediate-release solid dosage forms for Class I and III compounds when they exhibit rapid or very rapid dissolution (2-4). Class I includes drugs with high solubility and high permeability, whereas, Class III includes drugs with high solubility and low permeability. Class I drugs may receive a waiver if $85 \%$ or more of drug content is released in 15 min (very rapid release) in three different buffer solutions or may need to be compared using $f_{2}$ statistics when greater than $85 \%$ is released in $30 \mathrm{~min}$ (rapid release) $(4,7)$. Whereas, Class III drugs may receive a waiver only if $85 \%$ or more of drug content is released in $15 \mathrm{~min}$ (very rapid release) in three different buffer solutions $(4,7)$. The diagram in Figure 1 shows the steps 
for obtaining a biowaiver for immediate-release solid oral dosage forms containing BCS Class I and III drugs.

This study compares the dissolution results for different generic products containing BCS Class I drugs (fluoxetine and linezolid) and a Class III drug (fluconazole) marketed in Egypt with those of the innovator products. The objective was to investigate whether the different immediate-release oral formulations of fluoxetine, linezolid, and fluconazole fulfill the requirements of $85 \%$ or more drug release in 15 or $30 \mathrm{~min}$ in all three buffers (pH 1.2, pH 4.5 and $\mathrm{pH}$ 6.8) using in vitro dissolution testing. Drug release was assessed for bioequivalence in accordance with the WHO requirements (3) for biowaivers for immediate-release solid oral dosage forms containing BCS Class I and III drugs.

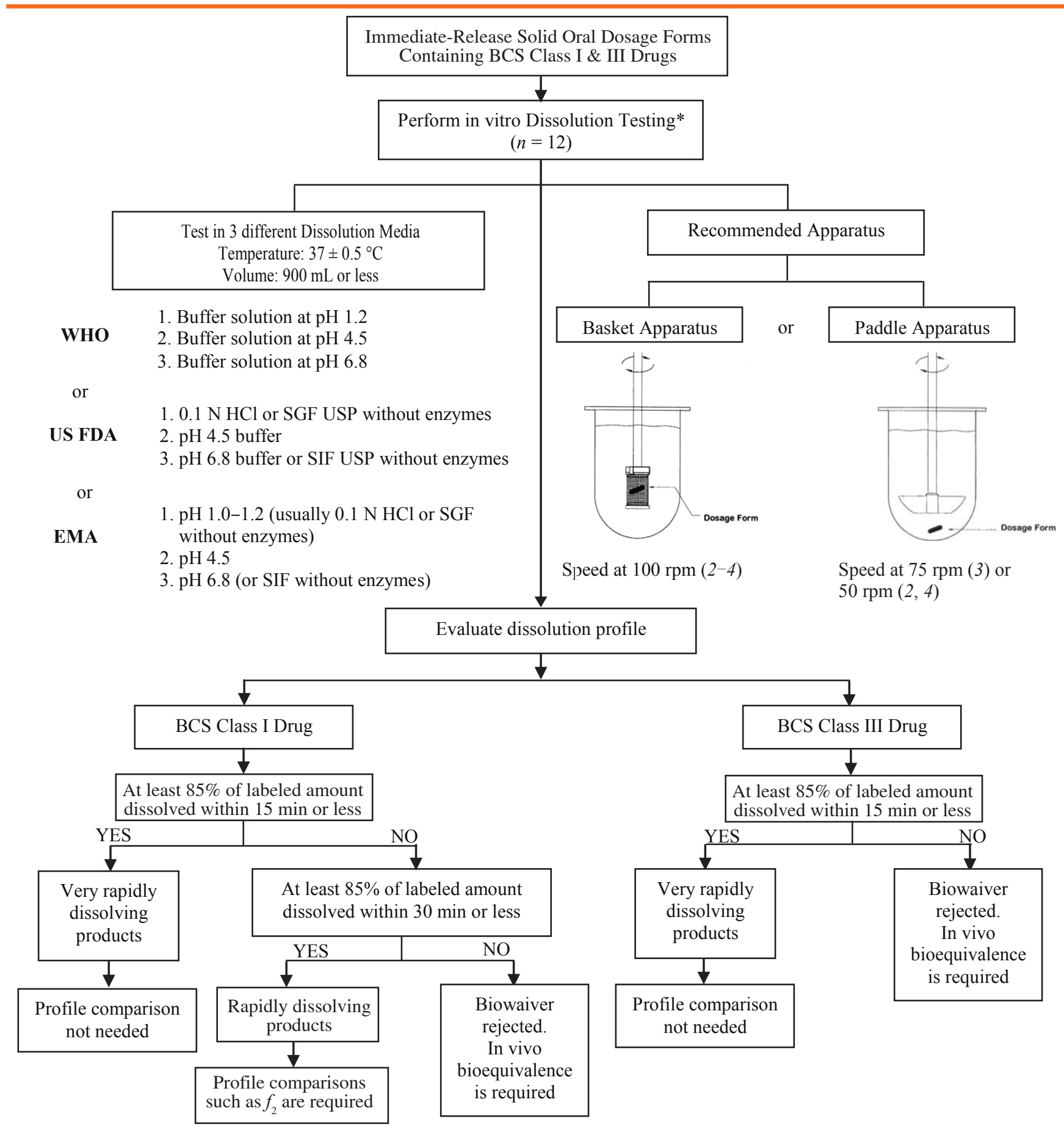

*Samples should be collected at a sufficient number of intervals to characterize the dissolution profile of the drug product SIF simulated intestinal fluid, SGF simulated gastric fluid

Figure 1. Flowchart for obtaining a biowaiver for immediate-release solid oral dosage forms containing BCS Class I and III Drugs (2-6). 


\section{MATERIALS AND METHODS}

\section{Reagents and Materials}

Fluoxetine $\mathrm{HCl}$ (purity 99\%), linezolid (purity 99\%), and fluconazole (purity 99\%) were purchased from SigmaAldrich Co. (St. Louis, MO, USA). Generic and innovator products were purchased from local pharmacies in Egypt. Buffer media for dissolution testing were prepared as per USP specifications at pH 1.2, 4.5, and 6.8 (11).

\section{Instrumentation and Equipment}

The $\mathrm{pH}$ of the prepared buffers was checked using a $\mathrm{pH}$ meter (Orion 520A, Waltham, MA, USA). A dissolution apparatus (Copley Scientific, Nottingham, UK) was used for dissolution testing of dosage forms. An ultrasonic bath (UltraSonik 57X, Elmsford, U.S.A) was used to dissolve the reference standards during preparation of standard solutions.

\section{Dissolution Testing}

Dissolution testing was performed at $\mathrm{pH} 1.2,4.5$, and 6.8 using a USP Apparatus 2 (paddle) dissolution apparatus (Copley Scientific, Nottingham, UK). The paddle speed was set at $75 \mathrm{rpm}$, and $900 \mathrm{~mL}$ of dissolution medium was used to test all samples. Prior to testing, the dissolution medium was preheated and degassed to prevent air bubble formation during transfer into the vessels. Dissolution testing was started after a temperature of $37 \pm 0.5{ }^{\circ} \mathrm{C}$ was confirmed in all vessels. Five-milliliter samples of the dissolution medium were withdrawn at appropriate time intervals $(1,2,3,4,5,10,15,30,45,60$, 90 , and $120 \mathrm{~min}$ ). Each sample was filtered through a $0.45-$ $\mu \mathrm{m}$ Millipore filter (Merck KGaA, Darmstadt, Germany), and fresh dissolution medium preheated to $37{ }^{\circ} \mathrm{C}$ was added to compensate for the withdrawn volume. Drug content was determined spectrophotometrically (Thermo Scientific Evolution 300 UV-vis Spectrophotometer, Madison, WI, USA). Each result represented an average of twelve measurements, and the error was expressed as standard deviation (SD). The cumulative amount and percentage of drug release at each sampling time was computed, and release profiles were plotted as the cumulative percentage of drug released versus time.

\section{Analytical Quantification}

Drug content was determined at $230 \mathrm{~nm}, 254 \mathrm{~nm}$, and 210 $\mathrm{nm}$ for fluoxetine, linezolid, and fluconazole, respectively, in the three dissolution media with reference to a standard curve constructed for each dissolution medium. Prior to sample testing, the spectrophotometric method was validated for its suitability in all three dissolution media for linearity, precision, and accuracy according to the USP General Chapter <1225> Validation of Compendial
Procedures (12). The linear range was selected based on an expected highest release concentration of $120 \%$ of drug content dissolved in $900 \mathrm{~mL}$ of each dissolution medium at $\mathrm{pH} 1.2,4.5$, and 6.8 for all the studied drugs. The lowest release concentrations were $1.2 \%, 2.3 \%$, and $6.5 \%$ for fluoxetine, linezolid, and fluconazole, respectively. The observed correlation coefficient was $r^{2}=0.999$ for all the studied products. The precision and accuracy of the assay method in all the three media were 1.3-8.1\% and $97.9-103 \%$, respectively, for fluoxetine; 0.9-4.3\% and $98.6-104 \%$, respectively, for linezolid; and $1.1-9.1 \%$ and $99.3-104 \%$, respectively, for fluconazole.

\section{Comparison of Release Profiles}

Fit Factors

A simple model-independent approach that uses fit factors was applied to compare the dissolution profiles of the selected drugs (13). Fit factors were adopted by the FDA Center for Drug Evaluation and Research (CDER), and the similarity factor was adopted by the European Medicines Evaluation Agency (EMA) Committee for Proprietary Medicinal Products (CPMP) as an assessment criterion of similarity between two in vitro dissolution profiles $(3,14,15)$. They include a difference factor $\left(f_{1}\right)$ and a similarity factor $\left(f_{2}\right)$.

The difference factor, $f_{1}$, shows the percentage error between the two curves at all time points. The similarity factor, $f_{2}$, as defined by and EMA (14) and FDA (15) is the logarithmic transform of the sum of the squares of the errors calculated from the difference between the test and the standard samples at all time points. The values of $f_{1}$ and $f_{2}$ for the generic products versus the reference were calculated from the means of the percentage of drug dissolved at each time point using:

$$
\begin{gathered}
f_{1}=\frac{\sum_{j=1}^{n}\left|R_{j}-T_{j}\right|}{\sum_{j=1}^{n} R_{j}} \times 100 \\
f_{2}=50 \times \log \left\{\left[1+\frac{1}{n} \sum_{j=1}^{n}\left(R_{j}-T_{j}\right)^{2}\right]^{-0.5} \times 100\right\}
\end{gathered}
$$

where $n$ is the number of time points and $R_{j}$ and $T_{j}$ are the percentages of reference and test product, respectively, released into the dissolution medium at time $j$.

According to the FDA guidance (4), dissolution profiles are similar if $f_{1}$ values are between 0 and 15 and $f_{2}$ values are between 50 and 100 . 
Additionally, the dissolution data of each product were used to calculate the model-independent parameters of mean dissolution time (MDT) (16) and dissolution efficiency (DE) (17).

\section{Dissolution Efficiency}

$D E$ was employed to compare drug release from various brands (18). DE is the area under the dissolution curve (AUC) between times $t_{1}$ and $t_{2}$ expressed as a percentage of the curve at maximum dissolution, $y_{100}$, over the same time $(17,19)$. $D E$ can be calculated with:

$$
D E=\frac{\int_{t_{1}}^{t_{2}} y \cdot d t}{y_{100} \times\left(t_{2}-t_{1}\right)} \times 100
$$

where $y$ is the percentage of dissolved product.

The integral of the numerator (i.e., $A U C$ ) is calculated by trapezoidal method (20). AUC is the sum of all the trapezoids and calculated by:

$$
A U C=\sum_{i=1}^{i=n} \frac{\left(t_{1}-t_{i-1}\right)\left(y_{i-1}+y_{i}\right)}{2}
$$

where $t_{i}$ is the $i^{\text {th }}$ time point and $y_{i}$ is the percentage of dissolved product at time $t_{i}(20)$. The reference and the test product are equivalent if the difference between their $D E$ values are within appropriate limits ( $\pm 10 \%)$.

\section{Moments of the Dissolution Profiles}

$M D T$ has been used to test the equivalence of two dissolution profiles $(16,21)$ or to compare different profiles statistically. MDT is calculated from the amount of drug released to the total cumulative drug $(16,18$, 22). Dissolution rate is expressed by MDT. The value of $M D T$ decreases with an increase in the release rate and is calculated from:

$$
M D T=\frac{\sum_{j=1}^{n} t_{j \text { mid }} \times \Delta M_{j}}{\sum_{j=1}^{n} \Delta M_{j}}
$$

where $j$ is the dissolution sample number, $n$ is the number of dissolution sample times, $t_{j \text { mid }}$ is the time at the midpoint between $t_{j}$ and $t_{j-1}$ (calculated as $t_{j}+t_{j-1} / 2$ ), and $\Delta M_{j}$ is the additional amount of drug dissolved between $t$ and $t_{j-1}$ (calculated as $M_{t}-M_{t-1}$ ). MDT calculations were carried out using the Excel add-in DDSolver (23).

\section{Statistical Analysis}

Comparisons among the generic products and the reference were made by the Student's $t$-test at the $95 \%$ confidence interval using Microsoft Office Excel 2007. Differences were considered significant if $p<0.05$.

\section{RESULTS AND DISCUSSION}

In vitro dissolution studies are sometimes used as an alternative to in vivo studies in assessing bioequivalence of immediate-release solid oral dosage forms containing BCS Class I and III drugs for both cost reduction and ethical considerations (24). In vitro tests embrace the principle that "No unnecessary human testing should be performed." The aim of this study was to evaluate the use of in vitro dissolution testing as a biowaiver procedure to assess the interchangeability of generics based on WHO recommendations (3). The dissolution process can be used as a surrogate for absorption $(25,26)$. Not all generic products containing the same drug in similar strengths and dosage forms are equivalent. Class I drugs with rapid dissolution and Class III drugs with very rapid dissolution may possess the same active ingredient and amount of drug but may show significant differences to in vitro equivalence requirements (26). Comparative in vitro dissolution should ensure the similarity of the test and comparator product in three different $\mathrm{pH}$ media considered relevant for absorption in the gastrointestinal tract (3).

The equivalence of the dissolution profiles of fluoxetine, linezolid, and fluconazole was assessed in terms of the fit factors $\left(f_{1}\right.$ and $\left.f_{2}\right)$, which were evaluated in accordance with the WHO requirements (3). Additionally, DE and MDT were also applied.

\section{Fluoxetine}

Fluoxetine belongs to BCS Class I (10). To establish bioequivalence using the in vitro method $(3,27)$, fluoxetine products should meet the biowaiver requirements presented in Figure 1. The dissolution profiles of fluoxetine marketed products versus the reference product are shown in Figure 2. The reference product $(\mathrm{R})$ and generic products (T1-T3) dissolved rapidly (85\% or more in 30 $\min$ ) in $\mathrm{pH} 1.2,4.5$, and 6.8. The three generic products met the $f_{1}$ and $f_{2}$ requirements (Table 1 ).

Dissolution profiles of all generic products were similar to dissolution profile of the reference product.

Fluoxetine release from the reference and generics was also assessed through $D E$ and $M D T$. If a drug has high $D E$, the API remains in contact with physiologic membranes for a long time and thus has high bioavailability in the assessed concentration range (28). MDT reflects the time for a drug to dissolve and is the first statistical moment for the cumulative dissolution process that provides an accurate drug release profile. The mean values of $M D T$ and $D E$ for the products under study are shown in Table 2. The MDT and $D E$ values for all generic 
products were similar to that of the reference product. The regulatory authorities (EMA, FDA, and WHO) accept rapidly dissolving (>85\% dissolved in $30 \mathrm{~min}$ ) BCS Class I drug products for biowaiver candidates. Accordingly, all the fluoxetine products would be considered in vitro equivalent in accordance with the biowaiver criteria.
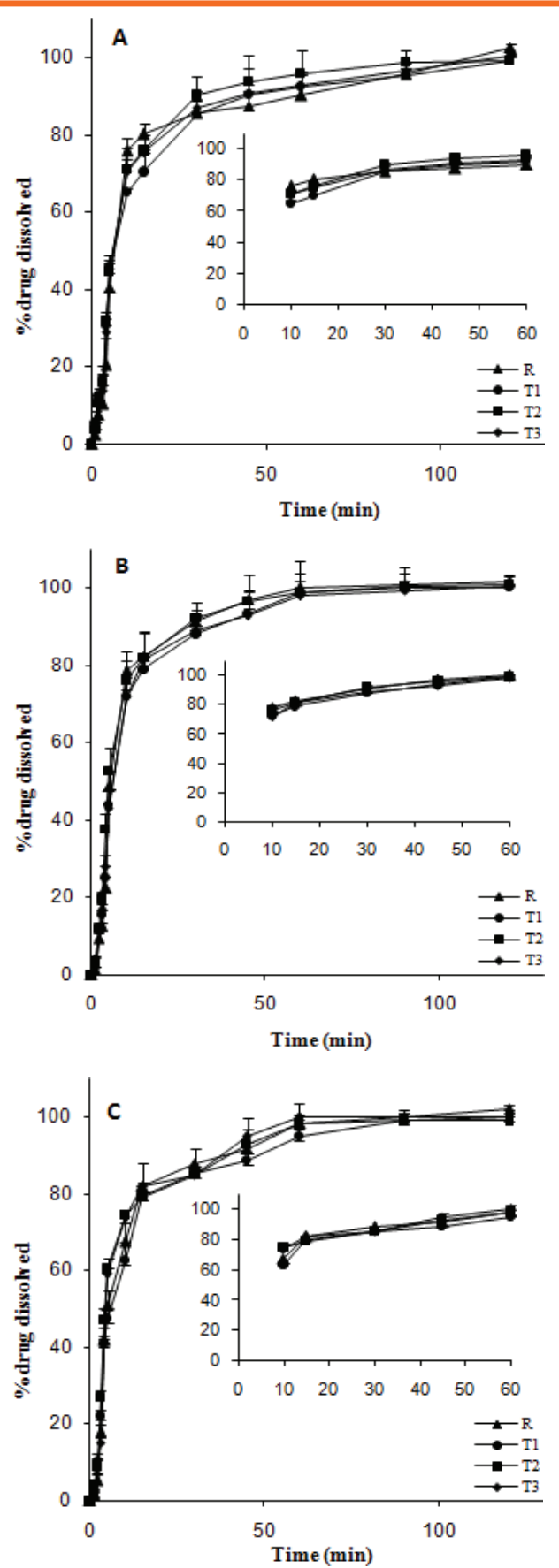

Figure 2. Dissolution profiles of various generic fluoxetine products in (A) $p H$ 1.2, (B) $p H 4.5$, and (C) $p H 6.8$.
Table 1. Comparison of Generic Fluoxetine, Linezolid, and Fluconazole Products

\begin{tabular}{|c|c|c|c|c|c|c|c|c|c|}
\hline \multirow[b]{2}{*}{ Product } & \multicolumn{3}{|c|}{ pH 1.2} & \multicolumn{3}{|c|}{ pH 4.5} & \multicolumn{3}{|c|}{ pH 6.8} \\
\hline & $\begin{array}{c}\% \\
\text { diss. }\end{array}$ & $f_{1}$ & $f_{2}$ & $\begin{array}{c}\% \\
\text { diss. }\end{array}$ & $f_{1}$ & $f_{2}$ & $\begin{array}{c}\% \\
\text { diss. }\end{array}$ & $f_{1}$ & $f_{2}$ \\
\hline \multicolumn{10}{|c|}{ Fluoxetine } \\
\hline$R$ & + & & & + & & & + & & \\
\hline $\mathrm{T} 1$ & + & 1.2 & 78.6 & + & 1.1 & 78.6 & + & 2.2 & 65.1 \\
\hline $\mathrm{T} 2$ & + & 4.6 & 51.0 & + & 4.3 & 51.4 & + & 3.6 & 54.8 \\
\hline T3 & + & 2.3 & 65.4 & + & 1.1 & 79.1 & + & 1.9 & 67.9 \\
\hline \multicolumn{10}{|c|}{ Linezolid } \\
\hline$R$ & ++ & & & ++ & & & ++ & & \\
\hline $\mathrm{T} 1$ & ++ & 0.3 & 93.3 & ++ & 0.4 & 91.7 & ++ & 0.5 & 90.1 \\
\hline $\mathrm{T} 2$ & ++ & 2.2 & 57.9 & ++ & 2.1 & 61.0 & ++ & 2.0 & 64.6 \\
\hline \multicolumn{10}{|c|}{ Fluconaole } \\
\hline$R$ & ++ & & & + & & & ++ & & \\
\hline $\mathrm{T} 1$ & ++ & 3.3 & 50.9 & + & 3.5 & 54.3 & ++ & 2.8 & 59.0 \\
\hline $\mathrm{T} 2$ & ++ & 2.3 & 58.0 & + & 0.9 & 81.2 & ++ & 1.8 & 67.9 \\
\hline T3 & ++ & 3.4 & 54.0 & - & 3.6 & 50.1 & - & 4.2 & $49.2^{*}$ \\
\hline
\end{tabular}

(+) At least $85 \%$ released within 30 min or less (rapidly dissolving)

$(++)$ At least $85 \%$ released within 15 min or less (very rapidly dissolving)

(-) Less than $85 \%$ released in more than $30 \mathrm{~min}$

(*) fails $f_{2}$

$f_{1}$ of $0-15$ ensures minor difference

$f_{2}>50$ represents similarity while $<50$ represent dissimilarity

Table 2. MDT and DE of Fluoxetine Products

\begin{tabular}{|c|c|c|c|c|c|c|}
\hline \multirow{2}{*}{ Product } & \multicolumn{2}{|c|}{ pH 1.2 } & \multicolumn{2}{c|}{ pH 4.5 } & \multicolumn{2}{c|}{ pH 6.8} \\
\cline { 2 - 7 } & $\begin{array}{c}\text { MDT } \\
\text { (min) }\end{array}$ & $\begin{array}{c}\text { DE } \\
\text { (\%) }\end{array}$ & $\begin{array}{c}\text { MDT } \\
\text { (min) }\end{array}$ & $\begin{array}{c}\text { DE } \\
\text { (\%) }\end{array}$ & $\begin{array}{c}\text { MDT } \\
\text { (min) }\end{array}$ & $\begin{array}{c}\text { DE } \\
(\%)\end{array}$ \\
\hline R & 6.2 & 86.8 & 5.6 & 92.3 & 6.0 & 90.5 \\
\hline T1 & 6.2 & 86.1 & 5.9 & 90.2 & 6.1 & 89.2 \\
\hline T2 & 5.8 & 89.3 & 5.4 & 92.2 & 5.4 & 90.0 \\
\hline T3 & 6.0 & 87.4 & 5.9 & 90.1 & 5.5 & 90.9 \\
\hline \multicolumn{7}{|c|}{ Linezolid } \\
\hline R & 1.7 & 98.8 & 2.9 & 96.8 & 4.2 & 94.7 \\
\hline T1 & 1.7 & 98.3 & 2.8 & 96.0 & 4.1 & 93.7 \\
\hline T2 & 2.2 & 98.7 & 3.3 & 95.8 & 4.5 & 92.9 \\
\hline \multicolumn{7}{|c|}{ Fluconaole } \\
\hline R & 2.2 & 97.7 & 5.3 & 92.1 & 5.1 & 90.8 \\
\hline T1 & 2.6 & 97.6 & 4.7 & 91.5 & 4.9 & 92.0 \\
\hline T2 & 2.5 & 96.4 & 5.7 & 92.9 & 5.4 & 89.4 \\
\hline T3 & 2.7 & 96.3 & $6.1^{\text {a }}$ & 88.6 & $5.9 a$ & 87.7 \\
\hline
\end{tabular}

${ }^{a}$ Significant difference from $R$ at $P<0.05$

\section{Linezolid}

All of the products ( $R, T 1$, and $T 2$ ) of linezolid (BCS Class I) dissolved very rapidly in $\mathrm{pH} 1.2,4.5$, and 6.8 , with a release of $>85 \%$ in $15 \mathrm{~min}$ in all three media. The dissolution 

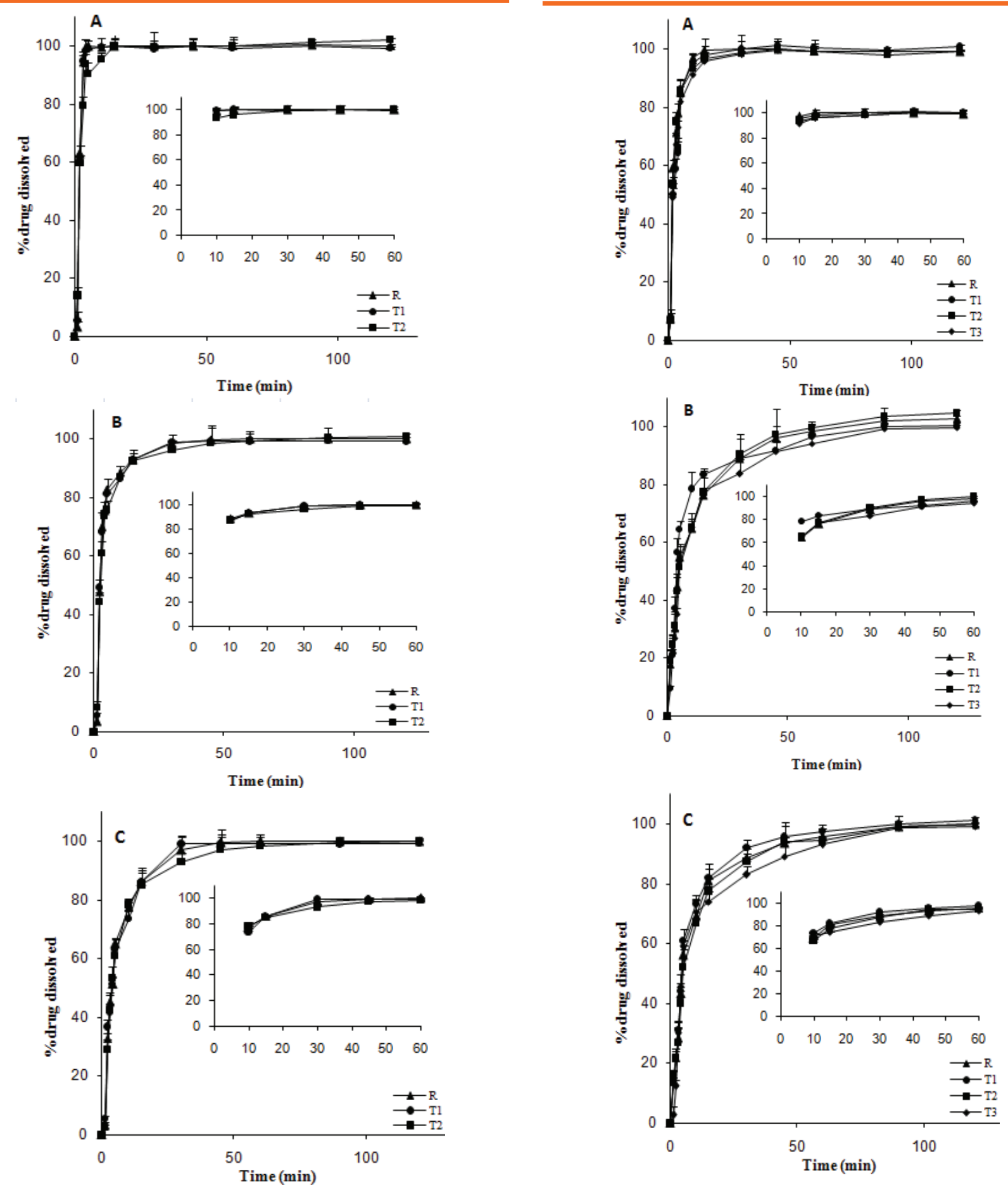

Figure 3. Dissolution profiles of various generic linezolid products in (A) $p H$ 1.2, (B) $p H 4.5$, and (C) $p H 6.8$.

profiles of marketed linezolid products versus the reference product are shown in Figure 3. The dissolution parameters shown in Tables 1 and 2 indicate the similarity of the studied generics to the reference product. None of the tested drugs exhibited $f_{2}$ outside of the established limits. Additionally, the results of $D E$ and $M D T$ support the conclusions drawn from the $f_{2}$ analysis. Therefore, all the linezolid products met the biowaiver criteria and would be considered in vitro equivalent. 
The reference and generic products (T1-T3) dissolved very rapidly in $\mathrm{pH} 1.2$ and met the requirement by exceeding a release of $85 \%$ fluconazole in $15 \mathrm{~min}$. In addition, they passed the $f_{2}$ requirement of $\geq 50$. In the case of $\mathrm{pH} 4.5$, all the products dissolved rapidly (85\% or more in $30 \mathrm{~min}$ ) except T3, for which more than $30 \mathrm{~min}$ was required to release $83.7 \%$ of fluconazole. On the other hand, $\mathrm{T} 1$ and $\mathrm{T} 2$ dissolved very rapidly in $\mathrm{pH} 6.8$ and thus met the requirement by exceeding a release of $85 \%$ in $15 \mathrm{~min}$. However, T3 required more than $30 \mathrm{~min}$ in $\mathrm{pH}$ 6.8 to release $81.1 \%$ of fluconazole, and the $f_{2}$ value in pH 6.8 was just below the acceptance value of 50 (49.2). Thus, T3 did not meet the biowaiver requirements for Class III drugs. The dissolution rate of $\mathrm{T} 3$ at $\mathrm{pH} 4.5$ and 6.8 was slower than that of the reference product, which is evidenced by the increased MDT values in $\mathrm{pH} 4.5$ (6.1 vs. $5.3 \mathrm{~min}$ ) and $\mathrm{pH} 6.8$ (5.9 vs. $5.1 \mathrm{~min})$. The reason for the poor dissolution of $\mathrm{T} 3$ at $\mathrm{pH} 4.5$ and 6.8 could be the variation of excipients or the manufacturing process. As a result, for generic products containing Class III drugs, the quality of excipients and the manufacturing process must allow full and timely release of the drug in the same way as the reference product at predefined conditions (29). Therefore, in vivo bioequivalence studies are required to ascertain the therapeutic equivalence.

\section{CONCLUSION}

In accordance with WHO recommendations for biowaivers for immediate-release solid oral dosage forms containing BCS Class I and III drugs, the interchangeability assessment of generics using in vitro dissolution similarity as a surrogate for in vivo bioavailability is a very simple approach and does not require the involvement of healthy volunteers in the study. This study used biowaiver conditions to assess the in vitro equivalence of some generic products containing BCS Class I and III drugs. All the BCS Class I generic products studied (fluoxetine and linezolid) met the biowaiver criteria and would be considered in vitro equivalent. For the BCS III drug (fluconazole), only one product (T3) did not meet the biowaiver requirements. However, all the products released fluconazole satisfactorily, with at least $80 \%$ of fluconazole dissolved within $30 \mathrm{~min}$. Pharmaceutical equivalence together with in vitro dissolution similarity could be considered a suitable surrogate to ensure the in vivo bioequivalence and hence the therapeutic equivalence of BCS Class I and III drugs.

\section{CONFLICT OF INTERESTS}

The authors declare no potential conflict of interest with respect to the research, authorship, or publication of this article.

\section{REFERENCES}

1. WHO Expert Committee on Specifications for Pharmaceutical Preparations. Multisource (generic) pharmaceutical products: guidelines on registration requirements to establish interchangeability; WHO Technical Report Series, No. 937, Annex 7; World Health Organization: Geneva, 2006.

2. Verbeeck, R. K.; Musuamba, F. T. The Revised 2010 EMA Guideline for the Investigation of Bioequivalence for Immediate Release Oral Formulations with Systemic Action. J. Pharm . Pharm. Sci. 2012, 15 (3), 376-388. DOI: 10.18433/J3VC8J.

3. WHO Prequalification Team-Medicines (PQTm). General notes on Biopharmaceutics Classification system (BCS)-based biowaiver applications; WHO Guidance Document; World Health Organization: Geneva, Switzerland, 2014.

4. Waiver of In Vivo Bioavailability and Bioequivalence Studies for Immediate-Release Solid Oral Dosage Forms Based on a Biopharmaceutics Classification System; Guidance for Industry; U.S. Department of Health and Human Services, Food and Drug Administration, Center for Drug Evaluation and Research (CDER), U.S. Government Printing Office: Washington, DC, 2000.

5. Löbenberg, R.; Amidon, G. L. Modern bioavailability, bioequivalence and biopharmaceutics classification system. New scientific approaches to international regulatory standards. Eur. J. Pharm. Biopharm. 2000, 50 (1), 3-12. DOI: 10.1016/ S0939-6411(00)00091-6.

6. Reddy, N. H.; Patnala, S.; Löbenberg, R.; Kanfer; I. In Vitro Dissolution of Generic Immediate-Release Solid Oral Dosage Forms Containing BCS Class I Drugs: Comparative Assessment of Metronidazole, Zidovudine, and Amoxicillin Versus Relevant Comparator Pharmaceutical Products in South Africa and India. AAPS PharmSciTech 2014, 15 (5), 1076-1086. DOI: 10.1208/ s12249-014-0135-6.

7. WHO Expert Committee on Specifications for Pharmaceutical Preparations, Fortieth Report; WHO Technical Report Series, No. 937; World Health Organization: Geneva, Switzerland, 2006.

8. Nightingale, S. L. Therapeutic Equivalence of Generic DrugsLetter to Health Practitioners, 1998. U.S. Food and Drug Administration Web site.

9. Gupta, E.; Barends, D. M.; Yamashita, E.; Lentz, K. A.; Harmsze, A. M.; Shah, V. P.; Dressman, J. B.; Lipper, R. A. Review of global regulations concerning biowaivers for immediate release solid oral dosage forms. Eur. J. Pharm. Sci. 2006, 29 (3-4), 315-324. DOI: 10.1016/j.ejps.2006.05.001.

10. BCS Database. Drug Delivery Foundation Web site. http://www. ddfint.org/bcs-database/ (accessed June 30, 2016).

11. Buffer Solutions. Pharmacopeia online Web site. http://www. uspbpep.com/usp29/v29240/usp29nf24s0_ris1s119.html. (accessed June 24, 2016).

12. <1225> Validation of Compendial Procedures. In The United States Pharmacopeia and National Formulary USP 36-NF 31; The United States Pharmacopeial Convention, Inc.: Rockville, MD, 2013. 
13. Moore, J. W.; Flanner, H. H. Mathematical Comparison of Curves with an Emphasis on In-Vitro Dissolution Profiles. Pharm. Technol. 1996, 20 (6), 64-74.

14. Guideline on the Investigation of Bioequivalence; CPMP/EWP/ QWP/1401/98 Rev. 1; Committee for Medicinal Products for Human Use (CHMP), European Medicines Agency: London, 2010.

15. Dissolution Testing of Immediate Release Solid Oral Dosage Forms; Guidance for Industry; U.S. Department of Health and Human Services, Food and Drug Administration, Center for Drug Evaluation and Research (CDER), U.S. Government Printing Office: Washington, DC, 1997.

16. Podczeck, F. Comparison of in vitro dissolution profiles by calculating mean dissolution time (MDT) or mean residence time (MRT). Int. J. Pharm. 1993, 97 (1-3), 93-100. DOI: 10.1016/03785173(93)90129-4.

17. Khan, K. A.; Rhodes, C. T. Effect of compaction pressure on the dissolution efficiency of some direct compression systems. Pharm. Acta Helv. 1972, 47 (10), 594-607.

18. Costa, P.; Sousa Lobo, J. M. Modeling and comparison of dissolution profiles. Eur. J. Pharm. Sci. 2001, 13 (2), 123-133. DOI: 10.1016/S0928-0987(01)00095-1.

19. Khan, K. A. The concept of dissolution efficiency. J. Pharm. Pharmacol. 1975, 27(1), 48-49. DOI: 10.1111/j.2042-7158.1975. tb09378.x.

20. Anderson. N. H.; Bauer, M.; Boussac, N.; Khan-Malek, R.; Munden, P.; Sardaro, M. An evaluation of fit factors and dissolution efficiency for the comparison of in vitro dissolution profiles. J. Pharm. Biomed. Anal. 1998, 17 (4-5), 811-822. DOI: 10.1016/S0731-7085(98)00011-9.

21. Brockmeier, D. In vitro/in vivo correlation of dissolution using moments of dissolution and transit times. Acta Pharm. Technol. 1986, 32, 164-174.

22. Costa, F. O.; Sousa, J. J. S.; Pais, A. A. C. C.; Formosinho, S. J. Comparison of dissolution profiles of Ibuprofen pellets. J. Controlled Release 2003, 89 (2), 199-212. DOI: 10.1016/S01683659(03)00033-6.

23. Zhang, Y.; Huo, M.; Zhou, J.; Zou, A.; Li, W.; Yao, C.; Xie, S. DDSolver: An Add-In Program for Modeling and Comparison of Drug Dissolution Profiles. AAPS J. 2010, 12 (3), 263-271. DOI: 10.1208/s12248-010-9185-1.

24. Polli, J. E. In Vitro Studies are Sometimes Better than Conventional Human Pharmacokinetic In Vivo Studies in Assessing Bioequivalence of Immediate-Release Solid Oral Dosage Forms. AAPS J. 2008, 10 (2), 289-299. DOI: 10.1208/ s12248-008-9027-6.

25. Saptarini, N. M. Evaluation of Content and Dissolution Profile of Generic Amoxicillin Tablets Marketed in Indonesia. Int. Res. J. Pharm. 2012, 3 (12), 64-66.

26. Villarroel Stuart, A.; Zuo, J.; Löbenberg, R. Investigating the Dissolution Profiles of Amoxicillin, Metronidazole, and Zidovudine Formulations used in Trinidad and Tobago, West Indies. AAPS PharmSciTech 2014, 15 (5), 1060-1069. DOI: 10.1208/s12249-014-0132-9.

27. WHO Expert Committee on Specifications for Pharmaceutical Preparations. Proposal to waive in vivo bioequivalence requirements for WHO Model List of Essential Medicines immediate-release, solid oral dosage forms; WHO Technical Report Series, No. 937, Annex 8; World Health Organization: Geneva, Switzerland, 2006.

28. de Freitas Santos, A., Jr.; Santos Barbosa, I.; Lima dos Santos, V.; Silva, R. L.; Caetite, E., Jr. Test of dissolution and comparison of in vitro dissolution profiles of coated ranitidine tablets marketed in Bahia, Brazil. Braz. J. Pharm. Sci. 2014, 50 (1), 83-89. DOI: 10.1590/S1984-82502011000100008.

29. Medina, J. R.; Salazar, D. K.; Hurtado, M.; Cortés, A. R.; Domínguez-Ramírez, A. M. Comparative in vitro dissolution study of carbamazepine immediate-release products using the USP paddles method and the flow-through cell system. Saudi Pharm. J. 2014, 22 (2), 141-147. DOI: 10.1016/j.jsps.2013.02.001. 